\title{
A Monadic Analysis of Information Flow Security with Mutable State
}

\author{
Karl Crary* $\quad$ Aleksey Kliger $\quad$ Frank Pfenning \\ Carnegie Mellon University
}

We explore the logical underpinnings of higher-order, security-typed languages with mutable state. Our analysis is based on a logic of information flow derived from lax logic and the monadic metalanguage. Thus, our logic deals with mutation explicitly, with impurity reflected in the types, in contrast to most higher-order security-typed languages, which deal with mutation implicitly via side-effects.

More importantly, we also take a store-oriented view of security, wherein security levels are associated with elements of the mutable store. This view matches closely with the operational semantics of low-level imperative languages where information flow is expressed by operations on the store. An interesting feature of our analysis lies in its treatment of upcalls (low-security computations that include high-security ones), employing an "informativeness" judgment indicating under what circumstances a type carries useful information.

\section{Introduction}

Security-typed languages use a type system to track the flow of information within a program to provide properties such as secrecy and integrity. Secrecy states that high-security information does not flow to low-security agents, and integrity dually states that low-security agents cannot corrupt high-security information. In this paper, we will restrict our attention to secrecy properties. A variety of security-typed languages have been proposed, and several of them are both higher-order (i.e., support first-class functions) and provide mutable state $[4,9,12,20]$.

However, when adopting one of these languages to the Typed Assembly Language [8] setting, one faces a tension between the high-level view of information flowing from the values of sub-terms to the result value of a complete term and the assembly-language imperative view of instructions

\footnotetext{
${ }^{*}$ This material is based on work supported in part by NSF grants CCR-9984812 and CCR-0121633. Any opinions, findings, and conclusions or recommendations in this publication are those of the authors and do not reflect the views of this agency.
}

Submitted for publication FCS'04. operating on a mutable store. What is needed is a typed calculus in which values have structure (i.e., like in high level languages) but information flows through the store (i.e., like in a low-level language).

In this paper, we explore this store-oriented view of information flow: one of the steps towards a TAL with information flow, we look at a language with a clean separation between values and computations. A suitable starting point is Moggi's monadic metalanguage [6, 7] and its corresponding logic (via a Curry-Howard isomorphism).

Our presentation of lax logic is based on that of Pfenning and Davies [10]. The principal distinctive feature of Pfenning and Davies's account is a syntactic distinction between terms and expressions, where terms are pure and expressions are (possibly) effectful. They show that this distinction allows the logic to possess some desirable properties (local soundness and local completeness) that state in essence that the logic's presentation is canonical. Although these properties are not particularly important here, the distinction also provides a clean separation between the pure and effectful parts of our analysis, which greatly simplifies our system.

Our system bears some resemblance to the work of Abadi et al. [1], who also use a monadic structure to reason about information flow. However whereas we use monads in a conventional manner to separate values from computations, they use a monad to endow values with a security level. It is not clear how to adopt their work to a low-level setting where the values and operations ought to correspond to those of a real machine.

A natural question is whether this store-oriented security discipline limits the expressive power of our account relative to ones based on a value-oriented discipline, but we show (in Section 5) that it does not.

Overview The static semantics of our analysis is based on two typing judgments, one for terms $(M)$ and one for expressions $(E)$. Recall that terms are pure and that security is associated with effects, so the typing judgment for terms makes no mention of security levels. Thus, the typing judgment takes the form $\Sigma ; \Gamma \vdash M: A$ (where $A$ is a type, $\Gamma$ is the usual context and $\Sigma$ assigns a type to the store).

Expressions, on the other hand, may have effects and therefore may interact with the security discipline. Each location in the store has a security level associated with it indicating the least security level that is authorized to read that location. Thus, the typing judgment for expressions tracks the security levels of all locations an expression reads or writes. Only the reads are of direct importance to the 
security discipline (recall that we do not address integrity), but writes must also be tracked since they provide a means of information flow. The judgment takes the form:

$$
\Sigma ; \Gamma \vdash E \div{ }_{(r, w)} A
$$

indicating that $r$ is an upper bound to the levels of $E$ 's reads, and $w$ is a lower bound to the levels of its writes, and also that $E$ has type $A$. Naturally we require that $r \sqsubseteq w$, or else $E$ could manifestly be leaking information.

So our language can be seen as a conservative extension of purely functional languages such as Haskell. Existing terms continue to be type-safe. On the other hand new effectful code that makes use of the security discipline can be cleanly separated.

In lax logic, expressions are internalized as terms using the monadic type $\bigcirc A$. Terms of type $\bigcirc A$ are suspended expressions of type $A$. Thus, the introduction form for the monadic type is a term construct, and the elimination form (which releases the suspended expression) is an expression construct. Similarly, our expressions are internalized as terms using a monadic type written $\bigcirc_{(r, w)} A$. Since the effects of the suspended expression will be released when the monad is eliminated, the levels of those effects must be recorded in the monad type.

Most of the rules in our account follow from the intuitions above. One remaining novelty deals with the information content of types. Ordinarily, an expression would be deemed to be leaking information if it were to read from a high-security location, use the result of the read to form a value, and pass that value to a low-security computation. However, that expression would not be leaking information if one could show that the type of that value contained no information, or contained information usable only by a high-security computation (who could have performed the read anyway). Thus the type system contains a judgment $\vdash A \nearrow a$ stating that the type $A$ contains information only for computations at the level $a$ at least. This notion of informativeness is essential to accounting for the key issue of upcalls (low-security computations that include high-security computations).

The remainder of this paper is organized as follows: In Section 2 we present our basic logical account, including static and dynamic semantics, but omitting the key issue of upcalls. In Section 3 we extend our account to deal with upcalls. In Section 4 we state and prove a non-interference theorem. In Section 5 we show that our store-oriented account provides at least the expressive power of value-oriented accounts by giving an embedding of the SLam calculus into our language. Section 6 discusses some related work, Section 7 offers some concluding remarks.

\section{The Secure Monadic Calculus}

We begin by describing the syntax, evaluation rules and an initial set of typing rules for our language. While this language will be secure, as we will see in the next section its type system rules out too many programs to be practical. By including some additional rules, we will be able to make it more useful while still retaining the required secrecy property.

As in other work on information flow, we have in mind an arbitrary fixed lattice (that is, a partial order $(\mathcal{L}, \sqsubseteq)$ equipped with a join $\sqcup$, meet $\sqcap$, and least $\perp$ and greatest
$T$ elements) of security levels. We use the meta-variables $a, b, c, r, w, \zeta$ to range over elements of $\mathcal{L}$.

Operation levels To track the flow of information, we classify expressions not only by the value that they return, but also by the security levels of their effects. In particular, we keep track of an operation level $o=(r, w)$, for each expression. The security level $r$ is an upper bound on the security levels of the store locations that the expression reads, while $w$ is a lower bound on the security level of the store locations to which it writes.

Since expressions that write at a security level below their read level may obviously be insecure, we restrict the operation levels to be elements of the set $\mathcal{O}=$ $\{(r, w) \in \mathcal{L} \times \mathcal{L} \mid r \sqsubseteq w\}$. Henceforth, when we write an operation level $(r, w)$, we will implicitly assume that it is an element of $\mathcal{O}$.

The operation levels have a natural ordering $(r, w) \preceq$ $\left(r^{\prime}, w^{\prime}\right)$. Given some expression $E$, if it reads from level at most $r$, then it surely reads from level at most $r^{\prime}$, provided that $r \sqsubseteq r^{\prime}$. Similarly, if it writes at level at least $w$, then it writes at level at least $w^{\prime}$, provided that $w^{\prime} \sqsubseteq w$. That is, operation levels are covariant in the reads and contravariant in the writes:

$$
(r, w) \preceq\left(r^{\prime}, w^{\prime}\right) \text { iff }\left(r \sqsubseteq r^{\prime} \text { and } w^{\prime} \sqsubseteq w\right)
$$

There is a subsumption principle for operation levels: if expression $E$ has operation level $o$, and $o \preceq o^{\prime}$, then $E$ has operation level $o^{\prime}$.

\subsection{Syntax}

The full syntax of our language is given in Figure 1. The language is split into two syntactic categories: terms $M$ and the expressions $E$, following Pfenning and Davies [10] . The terms are pure and evaluated to values $V$, while the expressions are executed for effect (but also return a value).

Terms At the term level, we have variables, unit, booleans and conditional terms, function abstractions and applications. For simplicity, we did not include a mechanism for defining recursive terms, although the inclusion of such a facility would not pose a problem. In support of our operational semantics, store locations are also terms. With each location $\ell$ is associated a fixed security level Level $(\ell)$. The store associates locations with the values they contain. A subtyping relation (explored later in this paper), allows us to treat store cells as either read-write, read-only, or writeonly.

The term val $E$ allows expressions to be included at the term level as an element of the monadic type $\bigcirc_{o} A$. Since terms are pure, a val $E$ does not execute the expression $E$, but rather represents a suspended computation.

Expressions The expressions include a trivial return expression $[M]$. The return expression has no effect, and simply returns the value to which $M$ evaluates. In general, when an expression has no read effects, we say its read level is $\perp$, and if an expression has no write effects, we say its write level is $\top$. Accordingly, the operation level of $[M]$ is $(\perp, \top)$. Note that $(\perp, \top)$ is the least element in the $\preceq$ ordering, so our subsumption principle will let us weaken the operation level of $[M]$ to any operation level. 


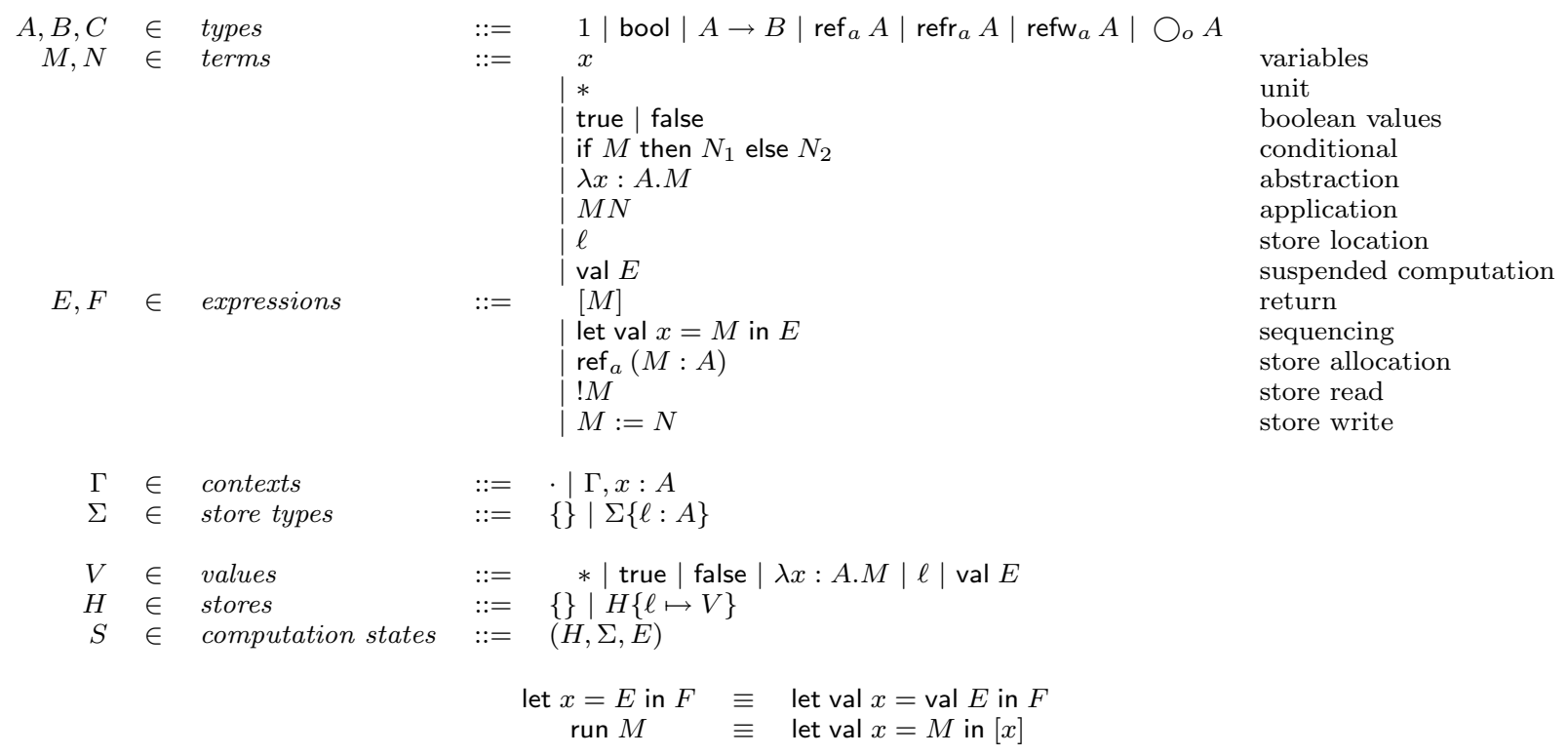

Figure 1: Syntax

The sequencing expression let val $x=M$ in $F$ evaluates $M$ down to some val $E$, and executes $E$ followed by $F$. The return value of expression $E$ is bound to the variable $x$ in $F$. If $E$ and $F$ both have operation level $o$, then so does the sequencing expression.

We will often write let $x=E$ in $F$ as syntactic sugar for let val $x=$ val $E$ in $F$, and run $M$ for let val $y=M$ in [y]. The derived typing rules are given in Appendix A.1.

In addition, there are expressions that allocate, read from, and write to the store. A read expression $! M$ has operation level $(a, \top)$, where $a$ is the security level of the store location being read, and returns the contents of the store location. Dually, a write expression $M:=N$ has operation level $(\perp, a)$ and updates the store location with the value of $N$; it does not return an interesting value (i.e., it returns unit).

Store allocation $\operatorname{ref}_{a}(M: A)$ specifies the security level $a$ and type $A$ of the new store location.

Allocation cannot leak information. Evidently, it is not a read operation. Less obviously, it is not a write operation either. With a write, another expression may learn something about the current computation by observing a change in the value stored at a particular store location. However, the key to this scenario is that the same location is mentioned by more than one expression. On the other hand, allocation creates a new location that is mentioned nowhere else. Thus, there can be no implicit flow of information via an allocation expression. As a result, allocation has operation level $(\perp, T)$. Of course if there were a primitive mechanism in place to distinguish one location from another (for example by comparing locations for equality), allocation would once again be observable.

Although there is not a primitive mechanism for recursion at the level of expressions, recursion can be encoded at the level of expressions using back-patching, see an example in Section 3.2.

States A computation state is a partially executed program, and consists of a triple $(H, \Sigma, E)$ of a store $H$, a store type $\Sigma$ and a closed expression $E$. The store maps locations to values, and the store type maps locations to the types of those values.

We assume that in a state $(H, \Sigma, E)$, the store binds occurrences of store locations $\ell$ in $H$ and $E$, and we identify computation states up to level-preserving renaming of store locations. In addition, as usual, we identify all constructs up to renaming of bound variables.

\subsection{Static Semantics}

The type system of our language consists of two main mutually recursive judgments for typing terms and expressions, and some judgments for typechecking stores, and computation states. The first judgment $\Sigma ; \Gamma \vdash M: A$ says that the term $M$ has type $A$ in the context $\Gamma$, where the store has type $\Sigma$. The second judgment typechecks expressions $\Sigma ; \Gamma \vdash E \div{ }_{o} A$ says that the expression $E$ returns a value of type $A$ and performs only operations within level $o$, as discussed above.

We assume that contexts $\Gamma$ are well-formed, that is, they contain at most one occurrence of each variable $x$. We tacitly rename bound variables prior to adding them to a context to maintain well-formedness. Similarly, we assume that store types are well-formed, that is, they contain at most one occurrence of each store location $\ell$.

Terms The typing rules for terms are unsurprising for a simply-typed lambda calculus with unit, abstraction and applications. A store location $\ell$ (provided that it is in $\operatorname{dom}(\Sigma)$ ) has a ref-type with its security level:

$$
\overline{\Sigma ; \Gamma \vdash \ell: \operatorname{ref}_{\text {Level }(\ell)} \Sigma(\ell)}
$$

A computation term val $E$ has the type $\bigcirc_{o} A$, provided the expression $E$ has type $A$ and operation level $o$ :

$$
\frac{\Sigma ; \Gamma \vdash E \div{ }_{o} A}{\Sigma ; \Gamma \vdash \operatorname{val} E: \bigcirc_{o} A}
$$

The remaining rules for terms are given in Appendix A.1. 


$$
\begin{gathered}
\frac{\vdash A \leq B \quad a \sqsubseteq b}{\vdash \operatorname{refr}_{a} A \leq \operatorname{refr}_{b} B}(8) \quad \frac{\vdash B \leq A \quad b \sqsubseteq a}{\vdash \operatorname{refw}_{a} A \leq \operatorname{refw}_{b} B} \\
\frac{\vdash A \leq B \quad a \sqsubseteq b}{\vdash \operatorname{ref}_{a} A \leq \operatorname{refr}_{b} B}(10) \quad \frac{\vdash B \leq A \quad b \sqsubseteq a}{\vdash \operatorname{ref}_{a} A \leq \operatorname{refw}_{b} B} \\
\frac{\vdash A \leq B \quad o \preceq o^{\prime}}{\vdash \bigcirc_{o} A \leq \bigcirc_{o^{\prime}} B}
\end{gathered}
$$

Figure 2: Selected subtyping rules.

Expressions The typing rules for expressions follow our informal description. Trivial computations have the type of their return value, and operation level $(\perp, \top)$ :

$$
\frac{\Sigma ; \Gamma \vdash M: A}{\Sigma ; \Gamma \vdash[M] \div(\perp, \top) A}
$$

The sequencing expression is well-typed provided both of the sub-computations have the same operation level (which may require using rule (6), below - the weakening rule for operation levels):

$$
\frac{\Sigma ; \Gamma \vdash M: \bigcirc_{o} A \quad \Sigma ; \Gamma, x: A \vdash E \div{ }_{o} A}{\Sigma ; \Gamma \vdash \text { let val } x=M \text { in } E \div{ }_{o} A}
$$

Allocation returns a new read/write store location:

$$
\frac{\Sigma ; \Gamma \vdash M: A}{\Sigma ; \Gamma \vdash \operatorname{ref}_{a}(M: A) \div(\perp, \top) \operatorname{ref}_{a} A}
$$

For read and write expressions we only require that the corresponding store location is readable or writable, respectively:

$$
\frac{\Sigma ; \Gamma \vdash M: \operatorname{refr}_{a} A}{\Sigma ; \Gamma \vdash ! M \div{ }_{(a, T)} A}(4) \quad \frac{\Sigma ; \Gamma \vdash M: \operatorname{refw}_{a} A \quad \Sigma ; \Gamma \vdash N: A}{\Sigma ; \Gamma \vdash M:=N \div(\perp, a)}
$$

In general we may weaken the operation level of a computation (indeed, as noted above, this is often necessary for the letval typing rule to apply):

$$
\frac{\Sigma ; \Gamma \vdash E \div{ }_{o} A \quad o \preceq o^{\prime}}{\Sigma ; \Gamma \vdash E \div{ }_{o^{\prime}} A}
$$

Subtyping A subsumption rule allows us to weaken the type $A$ of a term $M$ or an expression $E$, provided $A$ is a subtype of $B$ :

$\frac{\Sigma ; \Gamma \vdash M: A \vdash A \leq B}{\Sigma ; \Gamma \vdash M: B}$

$$
\frac{\Sigma ; \Gamma \vdash E \div{ }_{o} A \quad \vdash A \leq B}{\Sigma ; \Gamma \vdash E \div{ }_{o} A}
$$

Selected subtyping rules are given in Figure 2. Readonly store cells are covariant in the type of their contents and in their security level, and dually write-only cells are contravariant in each. Read/write store cells are neither covariant nor contravariant, but may be weakened to readonly or write-only cells. Finally, the monadic type $\bigcirc_{o} A$ is covariant in the return value type and operation level.

Stores and states A store $H$ is well-typed with store type $\Sigma$, provided that each value $V_{i}$ in the store is well typed under $\Sigma$ and the empty context, where $\Sigma$ has the same domain as $H$

$$
\frac{\operatorname{dom}(\Sigma)=\left\{\ell_{1}, \ldots, \ell_{n}\right\} \quad \Sigma ; \cdot \vdash V_{i}: \Sigma\left(\ell_{i}\right) \text { for } 1 \leq i \leq n}{\vdash\left\{\ell_{1} \mapsto V_{1}, \ldots \ell_{n} \mapsto V_{n}\right\}: \Sigma}
$$

(Note that since $\Sigma$ appears on the left in the premise of the rule, it must be well-formed).

A computation state $(H, \Sigma, E)$ is well-typed provided that the store and the expression are each well-typed with the same store type:

$$
\frac{\vdash H: \Sigma \quad \Sigma ; \cdot \vdash E \div{ }_{o} A}{\vdash(H, \Sigma, E) \div{ }_{o} A}
$$

\subsection{Operational Semantics and Safety}

A computation state is called terminal if it is of the form $(H, \Sigma,[V])$. An evaluation relation $S \rightarrow S^{\prime}$ gives the smallstep operational semantics for computation states. We write $S \downarrow$ if for some terminal state $S^{\prime}, S \rightarrow^{*} S^{\prime}$. Since terms are pure and do not have an effect on the store, their evaluation rules may be given simply by the relation $M \rightarrow M^{\prime}$ (no store is required). The evaluation rules for terms are entirely standard for a call-by-value language and are omitted. The evaluation rules for expressions are given in Figure 3.

We write $M[N / x]$ and $E[N / x]$ for the capture-avoiding substitution of $N$ for $x$ in the term $M$ or expression $E$. We write $H\{\ell \mapsto V\}$ for finite map that extends $H$ with $V$ at $\ell$.

A computation state $S$ is stuck if it is not terminal and there is no $S^{\prime}$ such that $S \rightarrow S^{\prime}$.

Lemma 2.1 (Type Safety). If $\vdash S \div$ o $A$ then $S$ is not stuck.

We prove type-safety in the usual manner, using preservation and progress lemmas (see the companion technical report [2] for proofs).

Although we do not formally prove a non-interference theorem at this stage, it should be fairly clear that any welltyped computation state must have the non-interference property. Consider a simple two-element security lattice $\mathcal{L}=\{\perp \sqsubset \top\}$. Since an expression ! $\ell$ where $\operatorname{Level}(\ell)=\top$ will have an operation level $(T, T)$, and since there is no way to force the read part of the operation level down once it has been increased, any computation that contains a highsecurity read will be forced to have operation level $(T, T)$. As a result any well-typed computation state with operation level $(\perp, \perp)$ or $(\perp, T)$ cannot depend on the values in high-security store locations.

\section{Upcalls}

Although the approach discussed so far is secure, it falls short of a practical language. There is no way to include a computation that reads from the high-security store in a larger low security computation. In any program with a high security read, the read level of the entire program is pushed up. However, many programs that contain upcalls to high security computations followed by low security code are secure.

Consider the program let $z=P$ in $E$ where $P \div{ }_{(\top, T)} 1$ and $E$ has operation level $(\perp, \perp)$. As we argued in the introduction, $P$ does not leak information because 1 carries no information. Thus we would like to give the entire program the operation level $(\perp, \perp)$. However the type system we have presented so far would instead promote the operation level of $E$ and the entire program to $(T, T)$. 


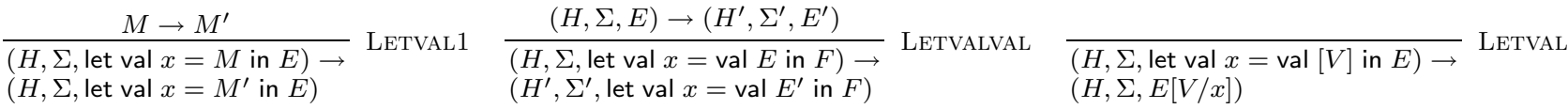

$$
\begin{aligned}
& \begin{array}{llll}
\frac{M \rightarrow M^{\prime}}{\left(H, \Sigma, \operatorname{ref}_{a}(M: A)\right) \rightarrow} \operatorname{ReF} 1 & \frac{\ell \notin \operatorname{dom}(H) \quad \operatorname{Level}(\ell)=a}{\left(H, \Sigma, \operatorname{ref}_{a}(V: A)\right) \rightarrow} \operatorname{REF} & \frac{M \rightarrow M^{\prime}}{(H, \Sigma, ! M) \rightarrow} \text { BANG1 } & \begin{array}{l}
(H, \Sigma, ! \ell) \rightarrow \\
\left(H, \Sigma, \operatorname{ref}_{a}\left(M^{\prime}: A\right)\right)
\end{array} \\
& (H\{\ell \mapsto V\}, \Sigma\{\ell: A\},[\ell]) & \left(H, \Sigma, ! M^{\prime}\right) & (H, \Sigma,[H(\ell)])
\end{array}
\end{aligned}
$$

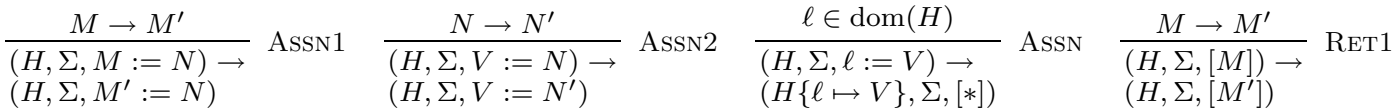

Figure 3: Operational Semantics (Expressions)

In order to have a logic of information flow, we must offer an account of upcalls. Indeed, the power to perform high security computations interspersed in a larger low-security computation is the sine qua non of useful secure programming languages. We offer a detailed analysis of two cases where upcalls do not violate our intuitive notion of security. From these examples, we develop a general principle for treating upcalls - our notion of informativeness discussed in Section 3.2. We take up the question of noninterference in Section 4.

\subsection{A more general example}

Now consider a computation $E$ with operation level $(r, w)$, but this time, suppose that $E$ has type $\operatorname{ref}_{a} B$ for some type $B$. Are there any situations where $E$ may be given a different operation level?

Suppose that $r \sqsubseteq a$. In that case, any computation that may read the $\operatorname{ref}_{a} B$ is also able to read any store locations that $E$ may read. Again, any computation can either do what $E$ does itself, or it cannot gain information from $E$ 's return value.

On the other hand, consider the case where $r \nsubseteq a$. The particular value of type $\operatorname{ref}_{a} B$ that $E$ returns may carry information from store locations at security level $r$. For example, $E$ may return one of two such store locations $\ell_{1}$ or $\ell_{2}$ from level $a$ based on some boolean value $V$ from a store location at security level $r$. In that case, a computation that reads at security level $a$ may learn something about $E$ 's reads (at level $r$ ) by reading from $E$ 's return value. Since $r \nsubseteq a$, this represents a violation of secure information flow.

So if $E$ returns a $\operatorname{ref}_{a} B$, we can demote its reading level whenever $r \sqsubseteq a$, because any computation that wishes to make use of that return value would need a read level of at least $r$. In other words, a ref ${ }_{a} B$ is informative only to computations that may read at least at some security level (namely $a$ ) above $r$.

Thus, we may wish to add a new rule for $\operatorname{ref}_{a} B$ :

$$
\frac{\Sigma ; \Gamma \vdash E \div(r, w) \operatorname{ref}_{a} B \quad r \sqsubseteq a}{\Sigma ; \Gamma \vdash E \div(\perp, w) \operatorname{ref}_{a} B}(* *)
$$

However, instead we add a general rule that allows us to demote the reading level of an expression $E$ :

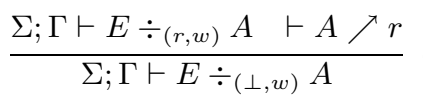

where the new informativeness judgment $\vdash A \nearrow r$ formalizes the idea that values of type $A$, if they are informative at all, are informative only at level $r$ or above. ${ }^{1}$

In terms of our new notation, our earlier observations are that $\vdash 1 \nearrow r$ for any $r$, and $\vdash \operatorname{ref}_{a} A \nearrow r$ whenever $r \sqsubseteq a$.

\subsection{Informativeness}

We now consider some properties of the new judgment $\vdash A \nearrow a$. Several structural rules for the judgment are immediate.

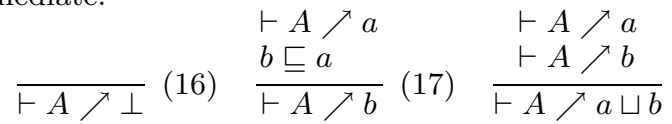

If $A$ is informative at all, then it's informative only at $\perp$ or above.

Also, if $A$ is informative only at or above $a$ and if $b \sqsubseteq$ $a$, then $A$ is informative only at or above $b$. That is, we may choose to discard some knowledge about when a type is informative

Finally, suppose $A$ is informative only above $a$, and $A$ is informative only above $b$. Then for any $r$ if values of type $A$ are informative to computations that read at $r$, we know that both $a \sqsubseteq r$ and $b \sqsubseteq r$. Therefore, for any such $r$, $a \sqcup b \sqsubseteq r$. So in fact, $A$ is informative only above $a \sqcup b$.

A value of type bool is informative for any computation at all, since it may be trivially analyzed with a conditional. So aside from the structural axiom $\vdash A \nearrow \perp$, there should be no other rules for bool. We would give a similar account of other type constructors that may be analyzed by cases. For example sum types $A+B$ or integers int.

A value of type $A \rightarrow B$ is used by applying it to some value and using the result. So $A \rightarrow B$ is informative exactly when $B$ is:

$$
\frac{\vdash B \nearrow a}{\vdash A \rightarrow B \nearrow a}
$$

We have already alluded to one rule for the type $\operatorname{ref}_{a} A$ :

$$
\overline{\vdash \operatorname{ref}_{a} A \nearrow a}
$$

which, in combination with the structural rule (17) gives us the upcall rule (**) from Section 3.1.

\footnotetext{
${ }^{1}$ Informativeness is closely related to protectedness in DCC [1] and to the tampering levels of [5]. We discuss the relationship in Section 6.
} 
However there is another rule for refs. Even if a computation can read from a store location of type ref $_{b} A$ (i.e., its read level is above $b$ ), only if $A$ is informative at its operation level, can $\operatorname{ref}_{b} A$ be informative:

$$
\frac{\vdash A \nearrow a}{\vdash \operatorname{ref}_{b} A \nearrow a}
$$

Read-only store locations are useful only to computations that may read from them. Consequently, by an argument similar to the one for read-write store cells, we have the two rules:

$$
\frac{\vdash A \nearrow a}{\vdash \operatorname{refr}_{b} A \nearrow a}(22) \quad \overline{\vdash \operatorname{refr}_{b} A \nearrow b}
$$

For write-only store cells $\operatorname{refw}_{a} A$, we have to consider aliasing. One way that a computation may learn whether two store locations are aliases is by writing a known value to one of them, and then reading out the value from the other. Because of subtyping, if a lower-security computation has a store location $\ell$ of type $\operatorname{refr}_{a} A$, a value of type refw $A$ may be informative if the computation can read from (the seemingly unrelated) $\ell$. As a result, we have the following rule:

$$
\overline{\vdash \operatorname{refw}}{ }_{a} A \nearrow a
$$

Finally, consider the type $\bigcirc_{(r, w)} A$. A value of this type is informative both to computations that may read at least security level $w$ (that is, the level the suspended expression writes to), and to computations for which the type $A$ is informative:

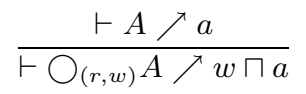

With informativeness in hand, many more useful terms become well-typed. Consider, for example, the term in Figure 4. The function untilFalse takes as argument a computation that reads and writes high before returning a boolean, and runs that computation repeatedly until it returns false. Recursion is accomplished using backpatching: a store location with a dummy value is allocated and is bound to wref, recursive calls in the body of the loop dereference wref and run the contents. The recursive knot is tied by overwriting the contents of wref with the real loop body $w$.

Interestingly, although untilFalse takes a high-security computation as an argument, our type system is able to

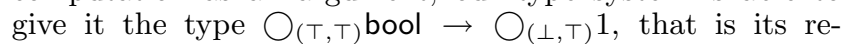
turn type is a low-security computation. Intuitively, even if $f$ is a high-security computation, untilFalse $f$ does not leak any information to low-security since any information gained from $f$ 's return value is used only within the loop. To formally show that untilFalse is well-typed, observe that $\Gamma \vdash$ let $b=$ run $c$ in run $(\ldots) \div(\top, \top) 1$, and since $\vdash 1 \nearrow \top$, it can be given operation level $(\perp, \top)$. The rest of the typing derivation is straightforward.

Having added all of these rules to the language, one may wonder whether the language is still secure. Because of the upcall rule (15), we can no longer argue that a computation with operation level $(r, w)$ does not read values at a security level above $r$ (it may now do so, provided the reads are not informative). So the simple informal argument at the end of Section 2 no longer applies. We take up the question of non-interference in the next section.

\section{Non-interference}

Informally, non-interference says that computations that have a low read level do not depend on values in high security store locations. As in similar arguments [20, 19], "low" means below some fixed security level $\zeta$, and "high" means not below $\zeta$.

Operationally, the low security sub-computations of a program should behave identically irrespective of the values in the high security store locations. On the other hand, it is alright for high security sub-computations to behave differently depending on values in high security store locations. However once a high security sub-computation completes, the low security behavior should again be identical modulo the parts of the computation state that are "out of view" of the low security part of the program.

Formally, we define an equivalence property of computation states such that two states are equivalent whenever they agree on the "in view" parts of the computation state. Then, in the style of a confluence proof, we show that this equivalence property is preserved under evaluation.

\subsection{Equivalence property}

We axiomatize the desired property as a collection of equivalence judgments on states, stores, terms and expressions.

Stores and States Certainly values in high security store locations are out of view. Less obviously, some values in the low security locations are out of view as well: if a low security store location appears only out of view, its value is also out of view. We parametrize the store equivalence judgment by a set $U$ of in view store locations. Two (welltyped) stores are equivalent only if their in view values are equivalent:

$$
\begin{array}{rc}
\vdash H_{1}: \Sigma_{1} & \Sigma_{1} \uparrow U=\Sigma_{2} \uparrow U \\
\vdash H_{2}: \Sigma_{2} & \Sigma_{1} ; \Sigma_{2} ; \cdot \vdash H_{1}(\ell) \approx_{\zeta} H_{2}(\ell): \Sigma_{1}(\ell) \\
& \text { for } \ell \in U
\end{array}
$$

Where the notation $\Sigma \uparrow X$ means $\Sigma$ restricted to locations in the set $X$.

For a pair of computation states, only low security locations that are common to both computations are in view. Since allocation does not leak information, it is possible for two programs to allocate different low security locations while executing high security sub-computations. However such locations are out of view for the low security subcomputation.

Pairs of computation states are equivalent if their stores are equivalent on the in-view locations, and if they have equivalent expressions:

$$
\begin{aligned}
& \vdash\left(H_{1}: \Sigma_{1}\right) \approx_{\zeta}^{\operatorname{dom}\left(H_{1}\right) \cap \operatorname{dom}\left(H_{2}\right) \cap \downarrow(\zeta)}\left(H_{2}: \Sigma_{2}\right) \\
& \frac{\Sigma_{1} ; \Sigma_{2} ; \cdot \vdash E_{1} \approx_{\zeta} E_{2} \div{ }_{o} A}{\vdash\left(H_{1}, \Sigma_{1}, E_{1}\right) \approx_{\zeta}\left(H_{2}, \Sigma_{2}, E_{2}\right) \div o A}
\end{aligned}
$$

Where $\downarrow(\zeta)=\{\ell \mid \operatorname{Level}(\ell) \sqsubseteq \zeta\}$ is the set of all low security locations. 


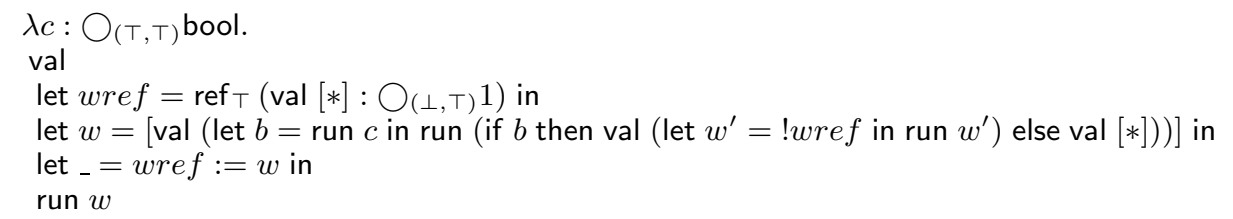

Figure 4: untilFalse $: \bigcirc_{(\top, T)}$ bool $\rightarrow \bigcirc_{(\perp, T)} 1$

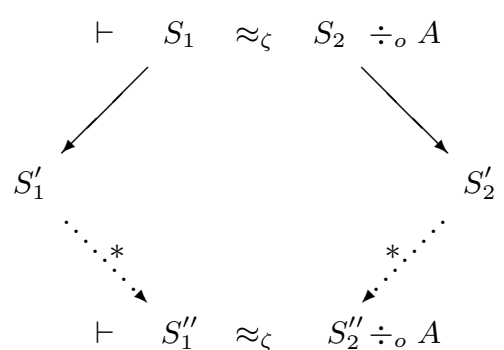

Figure 5: Hexagon Lemma

Terms and Expressions High security sub-computations of a program may return different values to the low security sub-computations. However, by the upcall rule, the type of those values must be informative only at high security.

Values of a type that is informative only at high security are out of view. As a result, any two values of such a type are equivalent since two such values vacuously agree on their in view parts:

$$
\frac{\Sigma_{1} ; \Gamma \vdash V_{1}: A \quad \Sigma_{2} ; \Gamma \vdash V_{2}: A \quad \vdash / a \quad a \nsubseteq \zeta}{\Sigma_{1} ; \Sigma_{2} ; \Gamma \vdash V_{1} \approx_{\zeta} V_{2}: A}
$$

The remaining rules for term and expression equivalence are congruence rules that merely require corresponding subterms or sub-expressions to be equivalent. They are given in the companion technical report.

\subsection{Non-interference theorem}

The main result necessary to establish non-interference is the so-called "Hexagon Lemma" (Figure 5): given two equivalent computations states that each take a step, we show that in zero or more steps we can reach two computation states that are again equivalent.

As previously noted, while a program is executing a high security sub-computation, it may behave differently based on the contents of the high-security store. However, as the following preliminary lemma shows, during any such high security steps, the in view parts of the stores remain equivalent.

Lemma 4.1 (High Security Step (HSS)). Given two states $\left(H_{1}, \Sigma_{1}, E_{1}\right)$ and $\left(H_{2}, \Sigma_{2}, E_{2}\right)$ such that

- $\vdash\left(H_{1}: \Sigma_{1}\right) \approx_{\zeta}^{U}\left(H_{2}: \Sigma_{2}\right)$ where $U=\operatorname{dom}\left(\Sigma_{1}\right) \cap \operatorname{dom}\left(\Sigma_{2}\right) \cap \downarrow(\zeta)$, and

- for $i=1,2$, there exist $C_{i}$ and $o_{i}=\left(r_{i}, w_{i}\right)$ such that $\Sigma_{i} ; \cdot \vdash E_{i} \div{ }_{o_{i}} C_{i}$ and $w_{i} \nsubseteq \zeta$.

$$
\begin{aligned}
& \text { If }\left(H_{i}, \Sigma_{i}, E_{i}\right) \rightarrow^{*}\left(H_{i}^{\prime}, \Sigma_{i}^{\prime}, E_{i}^{\prime}\right) \text { for } i=1,2 \text { then } \\
& \vdash\left(H_{1}^{\prime}: \Sigma_{1}^{\prime}\right) \approx_{\zeta}^{U^{\prime}}\left(H_{2}^{\prime}: \Sigma_{2}^{\prime}\right) \\
& \text { where } U^{\prime}=\operatorname{dom}\left(\Sigma_{1}^{\prime}\right) \cap \operatorname{dom}\left(\Sigma_{2}^{\prime}\right) \cap \downarrow(\zeta)
\end{aligned}
$$

The proof of this lemma appears in the companion technical report [2].

Lemma 4.2 (Hexagon Lemma). For all $\zeta$, if $o=(r, w)$ with $r \sqsubseteq \zeta$, and if $\vdash S_{1} \approx_{\zeta} S_{2} \div{ }_{o} C$ and $S_{1} \rightarrow S_{1}^{\prime}, S_{2} \rightarrow S_{2}^{\prime}$ where $\overline{S_{1}^{\prime}} \downarrow$ and $S_{2}^{\prime} \downarrow$ then there exist $S_{1}^{\prime \prime}, S_{2}^{\prime \prime}$ such that

$$
S_{1}^{\prime} \rightarrow^{*} S_{1}^{\prime \prime}, S_{2}^{\prime} \rightarrow{ }^{*} S_{2}^{\prime \prime} \text { and } \vdash S_{1}^{\prime \prime} \approx_{\zeta} S_{2}^{\prime \prime} \div{ }_{o} C
$$

Proof. By Inversion on $\vdash S_{1} \approx{ }_{\zeta} S_{2} \div{ }_{o} C$, we get that each computation state $S_{i}$ is a triple $\left(H_{i}, \Sigma_{i}, E_{i}\right)$, and that the two stores and the two expressions are equivalent, where $U=\operatorname{dom}\left(\Sigma_{1}\right) \cap \operatorname{dom}\left(\Sigma_{2}\right) \cap \downarrow(\zeta)$ is the set of in-view locations common to the two states:

$$
\begin{aligned}
& \vdash\left(H_{1}: \Sigma_{1}\right) \approx_{\zeta}^{U}\left(H_{2}: \Sigma_{2}\right) \\
& \Sigma_{1} ; \Sigma_{2} ; \cdot \vdash E_{1} \approx_{\zeta} E_{2} \div{ }_{o} C
\end{aligned}
$$

We prove the theorem by induction on latter derivation.

We consider one case below, the remaining cases are proved in the companion technical report.

$$
\text { Case: } \frac{\Sigma_{1} ; \Sigma_{2} ; \Gamma \vdash E_{1} \approx_{\zeta} E_{2} \div{ }_{\left(r^{\prime}, w\right)} C \vdash C \nearrow r^{\prime}}{\Sigma_{1} ; \Sigma_{2} ; \Gamma \vdash E_{1} \approx_{\zeta} E_{2} \div(\perp, w)}
$$

If $r^{\prime} \sqsubseteq \zeta$, we can invoke the induction hypothesis to get two equivalent computation states with operation level $\left(r^{\prime}, w\right)$, and then use the upcall rule to construct the desired derivation (with operation level $(r, w)$ ).

On the other hand, if $r^{\prime} \nsubseteq \zeta$, then since $r^{\prime} \sqsubseteq w$, it follows that $w \nsubseteq \zeta$ and so, by the High Security Step Lemma, running both of the computation states to completion produces equivalent stores. Since we also know that their return values are out of view, we can show that the resulting terminal states are equivalent.

Finally we are ready to prove non-interference. Starting with some initial store $H$ (well-typed with store type $\Sigma$ ) and an expression to execute $E$ with a free variable $x$, if we plug in different values $V_{1}, V_{2}$ for $x$, then provided that the in-view parts of $V_{1}, V_{2}$ are equivalent, we expect that if the resulting programs $\left(H, \Sigma, E\left[V_{1} / x\right]\right),\left(H, \Sigma, E\left[V_{2} / x\right]\right)$ run to termination, the resulting terminal states will be equivalent on their in view parts.

Theorem 4.3 (Non-interference). If $\vdash H: \Sigma$ and $\Sigma ; x: A \vdash E \div{ }_{(r, w)} B$ and if $\Sigma ; \Sigma ; \cdot \vdash V_{1} \approx_{r} V_{2}: A$ then if $\left(H, \Sigma, E\left[V_{1} / x\right]\right) \rightarrow^{*} S_{1}$ and $\left(H, \Sigma, E\left[V_{2} / x\right]\right) \rightarrow^{*} S_{2}$ and both $S_{1}, S_{2}$ are terminal, then $\vdash S_{1} \approx_{r} S_{2} \div{ }_{(r, w)} B$

Proof. By some easy structural properties, we can show that $\Sigma ; \Sigma ; \cdot \vdash E\left[V_{1} / x\right] \approx_{r} E\left[V_{2} / x\right] \div{ }_{(r, w)} B$. By repeated application of the Hexagon Lemma, the two computations evaluate to equivalent terminal states. Since the operational semantics are deterministic, those terminal states are $S_{1}$ and $S_{2}$, respectively. 


$$
\begin{array}{rlll}
t \in & \text { types } & ::= & 1 \mid \text { bool }\left|s_{1} \stackrel{\mathrm{pc}}{\longrightarrow} s_{2}\right| \text { ref } s \\
s & \in \text { security types } & ::= & (t, a) \\
b v & \in \text { base values } & ::= & * \mid \text { true } \mid \text { false }|\ell| \lambda[\mathrm{pc}] x: s . e \\
e & \in \text { expressions } & ::= & x\left|b v_{a}\right| \text { if } e_{1} \text { then } e_{2} \text { else } e_{3} \\
& & \left.\left|e_{1} e_{2}\right| \operatorname{ref}(e: s)\right)|! e| e:=e^{\prime}
\end{array}
$$

Figure 6: $\lambda_{\text {SEC }}^{\mathrm{REF}}$ Syntax

\section{Encoding a value-oriented language}

Our account differs substantially from prior secure programming languages where each value has a security level. In such languages, terms are classified by security types: pairs of an ordinary type and a security level. The type system ensures that each term is assigned a security level at least as high as the security level of the terms contributing to it. In our account only the store provides security. A natural question is whether we sacrifice expressive power in comparison to value-oriented secure languages.

We will show that our language is at least as expressive by exhibiting an embedding of a typical value-oriented imperative language. The embedding is not only type correct, but also preserves the security properties of the source language.

When a computation analyzes a value of a datatype by cases, each arm - by virtue of control flow - gains information about the subject of the case expression. In a purely functional setting, that additional information may only be used to compute the return value of the expression. Thus it suffices to require the return type of each arm (and thus the entire case expression) to be at least as secure as the case subject.

On the other hand, in an imperative setting, information gained via control-flow may leave an expression non-locally (e.g., via a write to the store). As a result, it becomes necessary to track such implicit flows of information. Secure imperative languages use a so-called program counter security level, pc, as a lower bound on the information that a computation may gain via control flow. Consequently, the results and effects of each expression must be at least as secure as any information gained via control flow.

In contrast to value-oriented secure programming languages, in our account we expect that case analysis is at the term level, and thus the arms of the case term do not have side-effects. We show that our approach is at least as expressive as imperative value-oriented secure languages.

We consider the language $\lambda_{\mathrm{SEC}}^{\mathrm{REF}}$ (summarized in Figure 6) of Zdancewic [16]. In addition to unit and boolean types, it has function types that are annotated with a lower bound on the write effects of the function body, and store locations. The base values of $\lambda_{\mathrm{SEC}}^{\mathrm{REF}}$ are annotated with a security level inside expressions.

The typing rules for $\lambda_{\mathrm{SEC}}^{\mathrm{REF}}$ base values are unsurprising, with the rule for lambda capturing the program counter annotation in the arrow type:

$$
\frac{\Sigma ; \Gamma, x: s[\mathrm{pc}] \vdash e: s^{\prime}}{\Sigma ; \Gamma \vdash \lambda[\mathrm{pc}] x: s . e: s \stackrel{\mathrm{pc}}{\longrightarrow} s^{\prime}}
$$

The rules for expressions are given in Figure 7.
Encoding In order to emulate the sealing behavior of valueoriented languages in our store-oriented discipline, we embed source-language values into read-only store cells in the target language. A slight complication arises in the translation of ref types since our language associates a security level with ref cells, but $\lambda_{\mathrm{SEC}}^{\mathrm{REF}}$ does not. In value-oriented security languages, the contents of ref cells have a security level, however. So we use the security level of the contents as the security level of the ref cell itself in our translation.

Function types are translated into functions types that return monadic types. In a $\lambda_{\mathrm{SEC}}^{\mathrm{REF}}$ function of type $s \stackrel{\mathrm{pc}}{\longrightarrow} s^{\prime}$ the program counter annotation pc is a conservative approximation of the information gained by the body of the function. Therefore, values written by the body must have security level at least pc. Thus, the corresponding writes in the translation must have write level at least pc. Consequently, the corresponding translated type for a function is $\bar{s} \rightarrow \bigcirc_{(\perp, \mathrm{pc})} \overline{s^{\prime}}$. The type encoding is (omitting base types):

$$
\begin{aligned}
\overline{(t, a)} & =\operatorname{refr}_{a} \bar{t} \\
\overline{\operatorname{ref}(t, a)} & =\operatorname{ref}_{a} \overline{(t, a)} \\
\overline{s_{1} \stackrel{\mathrm{pc}}{\longrightarrow} s_{2}} & =\overline{s_{1}} \rightarrow \bigcirc_{(\perp, \mathrm{pc})} \overline{s_{2}}
\end{aligned}
$$

The encoding for $\lambda_{\mathrm{SEC}}^{\mathrm{REF}}$ expressions is given by a pair of judgments $\Sigma ; \Gamma \vdash b v: t \Rightarrow M$ and $\Sigma ; \Gamma[\mathrm{pc}] \vdash e: s \Rightarrow E$, given in the companion technical report. The translation preserves typing, that is, if we extend the encoding ${ }^{\circ}$ pointwise to store types and to contexts, we have that whenever $\Sigma ; \Gamma[\mathrm{pc}] \vdash e: s \Rightarrow E$, it follows that $\bar{\Sigma} ; \bar{\Gamma} \vdash E \div \perp, \mathrm{pc} \bar{s}$. The proof appears in the companion technical report.

Non-interference Of course a type correct (but insecure) embedding could be constructed by ignoring the security levels of the source and placing everything at level $\perp$. We wish to show that the embedding is actually secure. To do so, we show that an instance of non-interference for $\lambda_{\mathrm{SEC}}^{\mathrm{REF}}$ is preserved by our translation.

Theorem 5.1 ( $\lambda_{\mathrm{SEC}}^{\mathrm{REF}}$ non-interference). Suppose $\Sigma_{0} ; x$ : $(t, a)[b] \vdash f:($ bool,$b) \Rightarrow F$ where $a \nsubseteq b$, and suppose that $H, \Sigma$ are such that $\Sigma \supseteq \overline{\Sigma_{0}}$, and $\vdash H: \Sigma$. If $\Sigma ; \cdot \vdash \ell_{i}: \operatorname{refr}_{a} \bar{t}$ for $i=1,2$ and if there exist $H_{1}, H_{2}, \Sigma_{1}, \Sigma_{2}, V_{1}, V_{2}$ such that

$$
\left(H^{\prime}, \Sigma^{\prime}, F\left[\ell_{i} / x\right]\right) \rightarrow^{*}\left(H_{i}, \Sigma_{i},\left[V_{i}\right]\right)
$$

for $i=1,2$ then $V_{i}=\ell_{i}^{\prime}$ and $H_{1}\left(\ell_{1}^{\prime}\right)=H_{2}\left(\ell_{2}^{\prime}\right)$ as booleans.

Proof. 1. From the type-correctness of the translation, and since the argument locations $\ell_{i}$ are out of view, by the non-interference theorem we conclude that $\vdash\left(H_{1}, \Sigma_{1},\left[V_{1}\right]\right) \approx_{b}\left(H_{2}, \Sigma_{2},\left[V_{2}\right]\right) \div(b, b) \quad \operatorname{refr}_{b}$ bool

2. By inversion and by a canonical forms lemma, each $V_{i}$ must be some store location $\ell_{i}^{\prime} \in \operatorname{dom}\left(\Sigma_{i}\right)$ and furthermore $\Sigma_{1} ; \Sigma_{2} ; \cdot \vdash V_{1} \approx_{b} V_{2}: \operatorname{refr}_{b}$ bool

3. By inversion on the latter equivalence, each $\Sigma_{i}\left(\ell_{i}^{\prime}\right)$ must either be out of view, or or $\ell_{1}^{\prime}=\ell_{2}^{\prime}$ with $\operatorname{Level}\left(\ell_{i}^{\prime}\right) \sqsubseteq$ $b$. But since $\Sigma_{i}\left(\ell_{i}^{\prime}\right)$ must be a subtype of $\operatorname{refr}_{b}$ bool, it cannot be out of view for a $b$-observer.

4. Therefore, $\ell_{1}^{\prime}=\ell_{2}^{\prime}$ are in the set of in-view locations $U=\operatorname{dom}\left(\Sigma_{1}\right) \cap \operatorname{dom}\left(\Sigma_{2}\right) \cap \downarrow(b)$, and by inversion on the store equivalence $\vdash\left(H_{1}: \Sigma_{1}\right) \approx_{\zeta}^{U}\left(H_{2}: \Sigma_{2}\right)$, the values in the respective stores must, in turn, be equivalent $\Sigma_{1} ; \Sigma_{2} ; \cdot \vdash H_{1}\left(\ell_{1}^{\prime}\right) \approx_{b} H_{2}\left(\ell_{2}^{\prime}\right)$ : bool 


$$
\begin{aligned}
& \frac{\Sigma ; \Gamma \vdash b v: t}{\Sigma ; \Gamma, x: s[\mathrm{pc}] \vdash x: s \sqcup \mathrm{pc}} \quad \frac{\Sigma ; \Gamma[\mathrm{pc}] \vdash e: s^{\prime} \quad \vdash s^{\prime} \leq s}{\Sigma ; \Gamma[\mathrm{pc}] \vdash b v_{a}:(t, a \sqcup \mathrm{pc})} \quad \frac{2 ; \Gamma[\mathrm{pc}] \vdash e: s}{}
\end{aligned}
$$

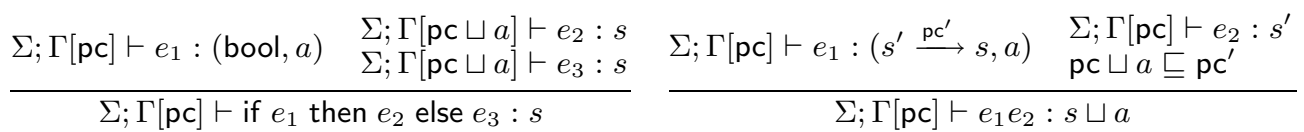

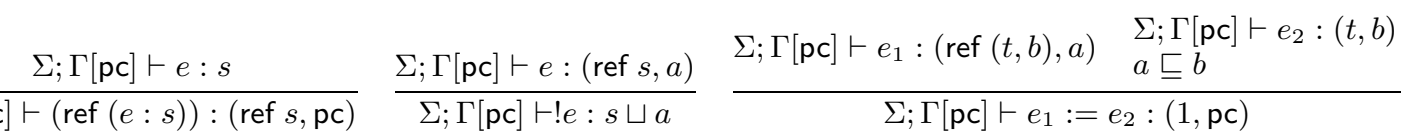

Figure 7: $\lambda_{\mathrm{SEC}}^{\mathrm{REF}}$ expression typing.

5. Since bool is informative at any security level, by inversion, it must be the case that $H_{1}\left(\ell_{1}^{\prime}\right)=H_{2}\left(\ell_{2}^{\prime}\right)$.

\section{Related Work}

There is a large body of existing work on type systems for secure information flow. Volpano, Smith and Irvine [15] first showed how to formulate an information flow analysis as a type system. An excellent survey by Sabelfeld and Myers [13] outlines the key ideas in the design of secure programming languages.

Prior work on secure languages with imperative features, such as Pottier and Simonet's work on core-ML $[11,12]$ take the side-effect view of computations: a term of any type $A$ may have a side-effect. In contrast, we have taken a monadic view of computation: only expressions may have an effect.

Our account is most related to the Dependency Core Calculus [1]. Like our language, DCC uses a family of monads to reason about information flow. However in DCC, terms of monadic type are used to seal up values at a security level. In our account, monads are used in a more traditional role as a means of threading state through a program.

Central to DCC is the notion of protectedness of a type at a security level. If $T$ is protected at $a$ then $T$ is at least as secure as $a$. This is closely related to our notion of informativeness.

When viewed through the lens of the encoding of (a pure subset of) $\lambda_{\mathrm{SEC}}^{\mathrm{REF}}$, the two relations serve the same purpose, ensuring that a computation's output is at least as secure as its inputs. In DCC, this is done directly. In our account, this occurs indirectly: to access a value carrying information only at a particular level, a computation must adopt a read level at least as high. (However, our account also offers the facility - not employed in the $\lambda_{\mathrm{SEC}}^{\mathrm{REF}}$ embedding - not to seal all computations' return values in order to obtain a $\perp$ effective read level).

The definitions of protectedness and informativeness are the same on the standard type operators, but do not include the idiosyncratic cases: our language has no analog of DCC's monad, nor does DCC contain references or a traditional (i.e., effects-oriented) monad. Moreover, if it did, we conjecture that DCC's definition for these would be somewhat different from ours.

Nevertheless, the similarity between the two suggests that our account might be profitably combined with DCC to produce a language capable of expressing security in both value-oriented and store-oriented fashions.

A further similarity exists between the tampering levels of Honda and Yoshida [5] and informativeness. They work in a concurrent setting of a typed $\pi$-calculus, and the tampering level of a process represents the least security level that may observe the effects of a process of a given type. They present a calculus in the style of [14] extended with local variables, reference types and higher-order procedures and a translation of it into their typed process calculus. Much of the complexity of their language stems from tracking the action set of a command, that is, the references (conflated with program variables) that a command may read or write. Our language may be seen as a restatement of their language in a more conventional monadic style. In addition, we exploit informativeness by dropping the read level of an expression when its result is not informative to low-level observers. In the setting of [5], that would correspond to leaving out the information that a command read from some variables from its action set whenever the command does not tamper below a certain security level.

Harrison et al. [3] observed that monads and monad transformers may be used to separate pieces of the state with different security levels, thus ensuring a kind of noninterference via the monad laws. However by construction their system does not allow computations to access any state with a different security level.

\section{Conclusion}

We give an account of secure information flow in the context of a higher-order language with mutable state. Moreover, motivated by a low-level store-oriented view of computation, we arrive at a view of security based on lax logic. Rather than sealing values at a security level, we instead associate security with the store. A family of monadic types is used to keep track of the effects of computations. To account for upcalls, we classify the informativeness of types at particular security levels.

Since we treat terms apart from the effectful expressions, our approach can straightforwardly encompass additional type constructors. The question of how to account for additional effects requires further work. From the point of view of non-interference, effects introduce the possibility of different behavior from seemingly related expressions. We expect that by further refining the monadic type to restrict 
the behavior of related terms, we may be able to account for effects such as I/O or non-local control transfers.

Certain complications beyond those discussed in this paper remain in developing a typed assembly language that tracks information flow. One problem to be dealt with is the re-use of registers between low-security and high-security computations. Since any mutation of a register by a high security computation could potentially be observed once it returns to a low-security caller. As a result it is necessary to exploit informativeness to ensure that the contents of registers are not informative to the caller. We conjecture that informativeness in conjunction with linear continuations [20] will prove invaluable to the design of a secure TAL.

Our formulation of the monadic language is in the style of Pfenning and Davies [10]. One avenue of future work is to study whether there is a formulation of information flow in a modal logic that decomposed our monad into the possibility and necessity modalities.

Acknowledgments Thanks to Matthew Harren and Steve Zdancewic for their comments and suggestions.

\section{References}

[1] M. Abadi, A. Banerjee, N. Heintze, and J. G. Riecke. A core calculus of dependency. In Twenty-Sixth ACM Symposium on Principles of Programming Languages, pages 147-160, San Antonio, Texas, Jan. 1999.

[2] K. Crary, A. Kliger, and F. Pfenning. A monadic analysis of information flow security with mutable state. Technical Report CMU-CS-03-164, Carnegie Mellon University, School of Computer Science, 2003.

[3] W. Harrison, M. Tullsen, and J. Hook. Domain separation by construction. In Foundations of Computer Security Workshop(FCS'03), Ottowa, Canada, June 2003.

[4] N. Heintze and J. G. Riecke. The SLam calculus: Programming with secerecy and integrity. In Twenty-Fifth ACM Symposium on Principles of Programming Languages, pages 365 - 377, San Diego, California, Jan. 1998.

[5] K. Honda and N. Yoshida. A uniform type structure for secure information flow. In Twenty-Ninth ACM Symposium on Principles of Programming Languages, pages 81-92, Jan. 2002.

[6] E. Moggi. Computational lambda-calculus and monads. In Fourth IEEE Symposium on Logic in Computer Science, pages 14-23, 1989.

[7] E. Moggi. Notions of computation and monads. Information and Computation, 93:55-92, 1991.

[8] G. Morrisett, D. Walker, K. Crary, and N. Glew. From System $\mathrm{F}$ to typed assembly language. $A C M$ Transactions on Programming Languages and Systems, 21(3):527-568, May 1999. An earlier version appeared in the 1998 Symposium on Principles of Programming Languages.

[9] A. C. Myers. JFlow: Practical mostly-static information flow control. In Twenty-Sixth ACM Symposium on Principles of Programming Languages, pages 228-241, San Antonio, Texas, Jan. 1999.
[10] F. Pfenning and R. Davies. A judgmental reconstruction of modal logic. Mathematical Structures in Computer Science, 11(4):511-540, 2001.

[11] F. Pottier and V. Simonet. Information flow inference for ML. In Twenty-Ninth ACM Symposium on Principles of Programming Languages, Jan. 2002.

[12] F. Pottier and V. Simonet. Information flow inference for ML. ACM Transactions on Programming Languages and Systems, 25(1):117-158, Jan. 2003.

[13] A. Sabelfeld and A. C. Myers. Language-based information-flow security. IEEE Journal on Selected Areas in Communications, 21(1):5 - 19, Jan. 2003. special issue on Formal Methods in Security.

[14] G. Smith and D. Volpano. Secure information flow in a multi-threaded imperative language. Twenty-Fifth ACM Symposium on Principles of Programming Languages, pages 355 - 364, 1998.

[15] D. Volpano, G. Smith, and C. Irvine. A sound type system for secure flow analysis. Journal of Computer Security, 4(3):167-187, 1996.

[16] S. Zdancewic. Programming Languages for Information Security. PhD thesis, Department of Computer Science, Cornell University, Ithaca, New York, 2002.

[17] S. Zdancewic. A type system for robust declassification. In Nineteenth Mathematical Foundations of Programming Semantics, Electronic Notes in Theoretical Computer Science, Mar. 2003.

[18] S. Zdancewic and A. C. Myers. Robust declassification. In Fourteenth IEEE Computer Security Foundations Workshop, pages 15 - 23, Cape Brenton, Nova Scotia, Canada, 2001.

[19] S. Zdancewic and A. C. Myers. Secure information flow and CPS. In Tenth European Symposium on Programming, volume 2028 of Lecture Notes in Computer Science, pages 46 - 61. Springer-Verlag, Apr. 2001.

[20] S. Zdancewic and A. C. Myers. Secure information flow via linear continuations. Higher Order and Symbolic Computation, 15(2-3):209-234, Sept. 2002.

\section{A Judgments}

\section{A.1 Typing judgment rules}

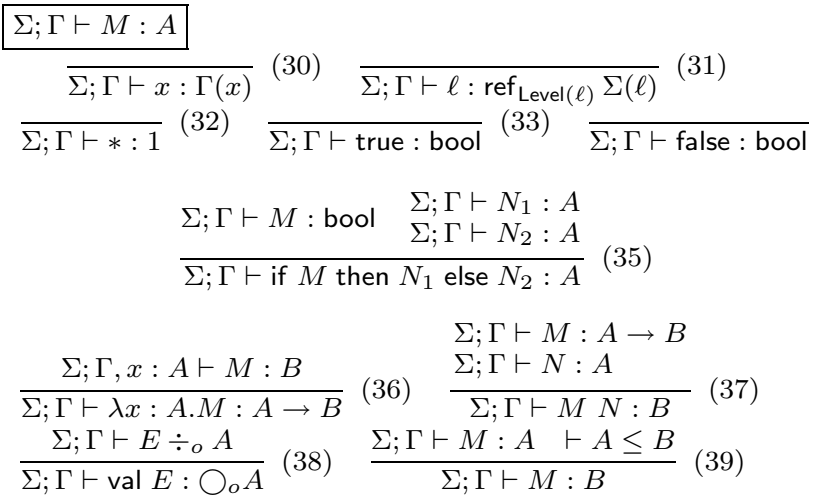

